\title{
A Model for Late-Stage Modification of Polyurethane Dendrimers Us- ing Thiol-Ene Click Chemistry
}

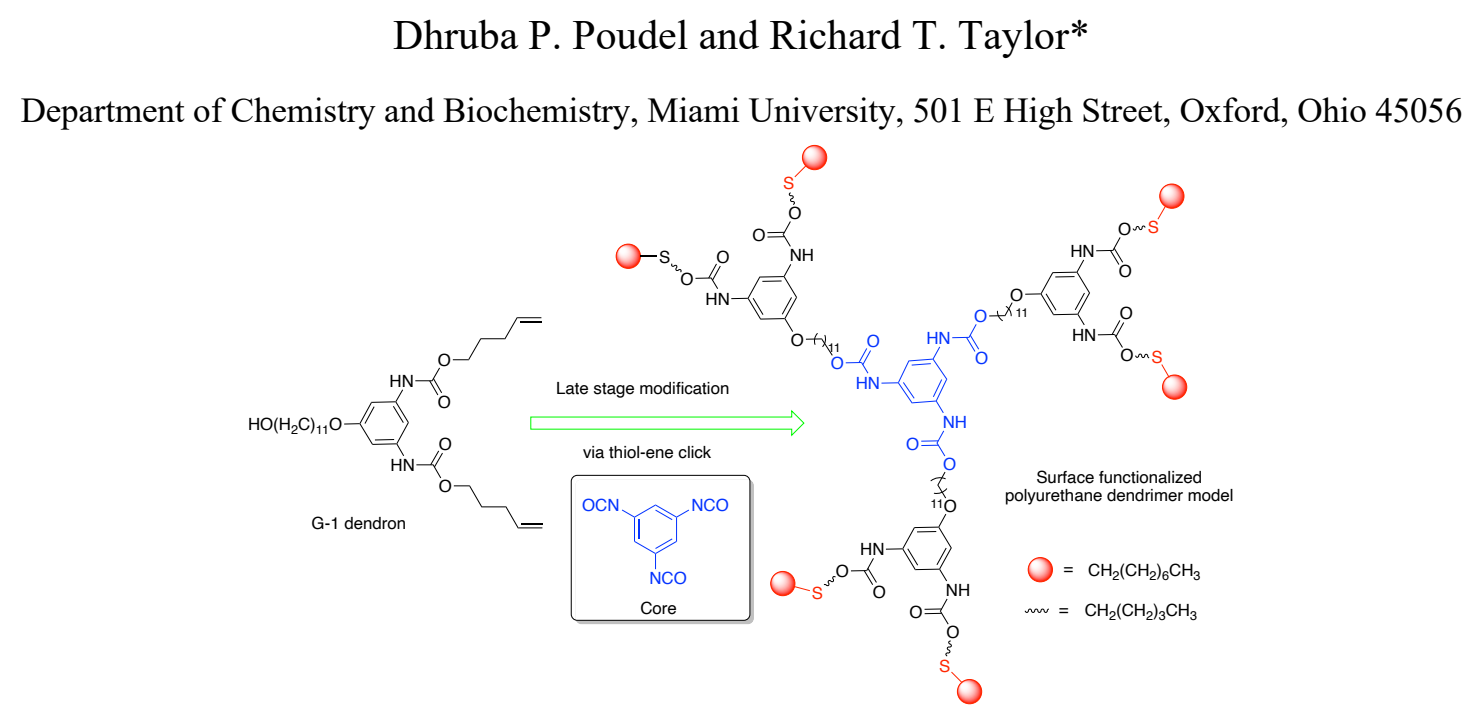

ABSTRACT: Protecting group free one-pot multicomponent Curtius reaction affords a robust and versatile $\mathrm{AB}_{2}$ type diurethane dendron, which ensures late-stage modification of both dendron and dendritic macromolecule yielding a surface functionalized polyurethane dendrimer. This strategy is highly useful in the synthesis of unsymmetrical or Janus dendrimers.

Since the first successful synthesis of poly(amidoamine) dendrimers by Tomalia et al. in $1985,{ }^{1}$ development of dendritic macromolecules, including dendrimers and hyperbranched polymers has developed rapidly in the field of macromolecular chemistry ${ }^{2}$ because of their extensive applications in the chemical ${ }^{3}$ and biomedical fields. ${ }^{4,5}$ Dendritic macromers have been reported for a wide variety of compounds like polyethers, polyamines, polyamides, polyarylenes, polycarbosilanes, and polycarbonates. Though a few such structures are reported in the field of polyurethanes, construction of a welldefined architecture of polyurethane dendrimers (PUDs) ${ }^{6}$ is challenging owing to high reactivity of externally added or insitu formed isocyanates towards nucleophiles, which could lead to significant amounts of side products. Pleasingly, two seminal works published simultaneously in 1993 by two research groups founded synthetic routes to PUDs, which are valid to date. The first route described by Spindler and Fréchet $^{7}$ using isocyanate chemistry assured the synthesis of dendritic structures via growth of two generations in a single synthetic operation. The second route described by Kumar and Ramakrishnan ${ }^{7}$ using Curtius rearrangement as an isocyanate free approach trapped in-situ formed isocyanate by an alcohol affording a urethane.

PUDs have been synthesized employing both divergent $t^{8-12}$ and convergent ${ }^{13-16}$ methods in the last two and half decades after the aforementioned pioneering works. First reported by Hawker and Fréchet, ${ }^{13}$ the convergent synthesis involves a small number reactions per molecule during the coupling of dendron and activation of functional group at focal point. This ensures greater structural control than in divergent synthesis appraoch. ${ }^{17}$ Moreover, the functional groups can be precisely placed throughout the dendritic structure, an attribute required to construct functional macromolecules. Nevertheless, fewer reports have been reported on PUDs employing the convergent method. Previously, our group reported convergent synthesis of PUDs containing dodecyl as end groups using a protection/deprotection strategy. ${ }^{18,19}$ This work reports on a fast, efficient, and protecting group free approach to the synthesis of PUDs where terminal pentene functionalized end groups of dendron can further undergo pre- or post-modification via thiol-ene click chemistry. This enables easy modification of the dendritic periphery, which could be of particular interest because these peripheral groups are the moieties to come in frequent contact with the external environment.

As a proof of concept, herein we report the synthesis of the first-generation dendritic wedge, its attachment to a core structure, and pre-and post-modification using thiol-ene reaction. The versatility of this approach is depicted by an $\mathrm{AB}_{2}$ type dendritic monomer that can undergo either a thiol-ene click reaction ${ }^{20}$ or attachment to the core. As shown in Scheme 1 'hydroxy' and 'ene' functionalized dendrons can be utilized in either of the two ways- click and attach to the core or attach to the core and click- to synthesize a polyurethane dendrimer. 
Scheme 1. General representation of strategy employed

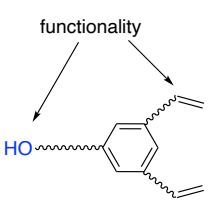

G-1 dendron
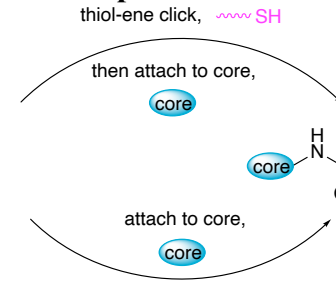

then thiol-ene click, mm SH

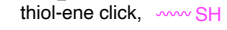

polyurethane dendrimer model

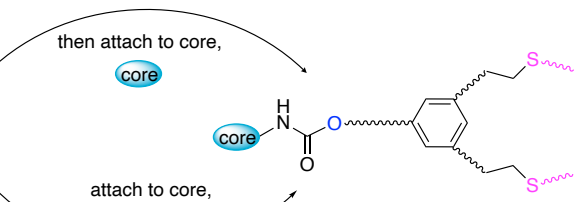

To build $\mathrm{AB}_{2}$ type dendron or branching monomer, we selected three molecules, 5-hydroxyisophthalic acid, 4-penten-1ol, and 11- bromoundecanol as branching unit, peripheral group, and spacer group respectively. Accordingly, the branching monomer was prepared in a two-step sequence of reactions. We exploited the Curtius reaction ${ }^{21}$ to synthesize the phenolic diurethane 1. The formation of $\mathbf{1}$ involved one-pot multicomponent Curtius reaction in which 5hydroxyisophthalic acid (1 eq) 1a was converted to an isocyanate analogue $\mathbf{1 b}$ through an acyl azide intermediate under mild condition using diphenyl phosphoryl azide ${ }^{22}$ (DPPA, 2.1 eq) and triethylamine (2 eq) (Scheme S2). Organic isocyanates are electrophilic reactive intermediates, which can be trapped easily by nucleophiles in situ thereby forming the urethane linkage. The hydroxy diisocyanate $\mathbf{1 b}$ was trapped by 4 penten-1-ol to afford the phenolic diurethane 1, which in turn furnished branching monomer $\mathbf{2}$ with an excellent yield when refluxed with 11-bromoundecanol. Unlike previously reported synthetic protocol, ${ }^{18,19}$ this strategy is concise and does not require any protection-deprotection of functional groups.

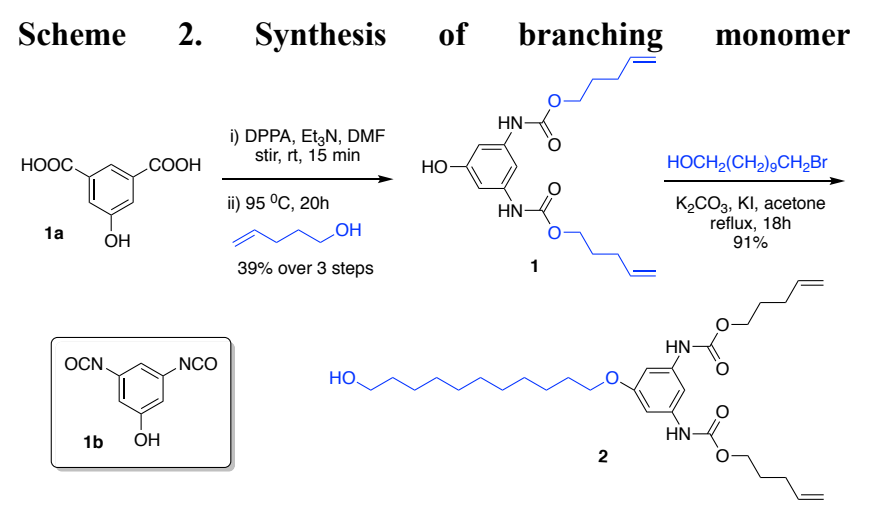

Protecting group-free Curtius reaction is a key step to from the urethane linkage in this approach and we spent some time investigating the efficacy of this reaction. This reduces an extra step required to activate the dendron at its focal point. Since the reaction intermediate $\mathbf{1 b}$ (Scheme 2) has nucleophilic phenolic group, it could potentially compete with 4penten-1-ol to react with its own isocyanate leading to the formation of polymeric side products. This directed us to optimize the reaction conditions. Unprotected phenolic hydroxy group in $\mathbf{1 b}$ resulted in two noticeable side products - diurethane phosphate 1c and dimer of monourethane 1d. Taking ad-

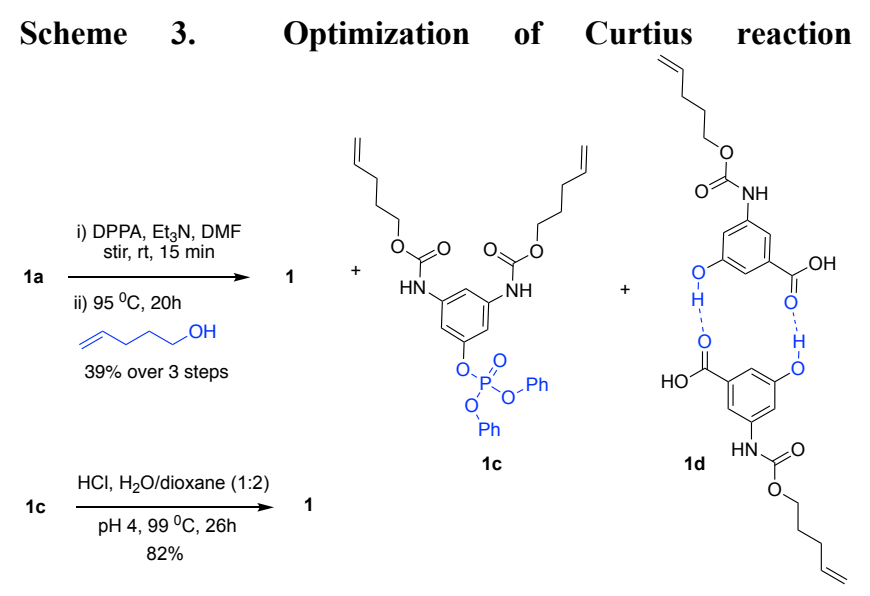

Table 1. Effect of base on Curtius reaction ${ }^{a}$

\begin{tabular}{|c|c|c|c|c|c|}
\hline \multirow{2}{*}{ Entry } & Base & pKa & \multicolumn{3}{|c|}{ Yield (\%) } \\
\cline { 3 - 6 } & & & 1 & $1 \mathrm{c}$ & $1 \mathrm{~d}$ \\
\hline $\mathbf{1}$ & Pyridine & 5.2 & 19 & 11 & 2 \\
\hline $\mathbf{2}$ & PVP & 5.6 & 5 & 11 & 36 \\
\hline $\mathbf{3}$ & DMAP & 9.6 & 20 & 12 & 5 \\
\hline $\mathbf{4}$ & $\mathrm{Et}_{3} \mathrm{~N}$ & 10.8 & 39 & 10 & 4 \\
\hline $\mathbf{5}$ & No base & - & 1 & 24 & 1 \\
\hline
\end{tabular}

${ }^{a}$ Conditions: 1a (1.0 eq), DPPA (2.1 eq), triethylamine (2.0 eq), 4- penten-1-ol (1.5 eq). Crude was purified by flash chromatography using ethyl acetate/hexane as eluting solvent.

vantage of difference between $\mathrm{pKa}$ values of aromatic hydroxy $(\sim 10)$ and carboxylic groups $(\sim 3-5)$, we anticipated that a base with $\mathrm{pKa}$ less than that of phenolic $-\mathrm{OH}(\sim 10)$ could prevent the potential formation of urethane phosphate. Surprisingly, the bases with low 


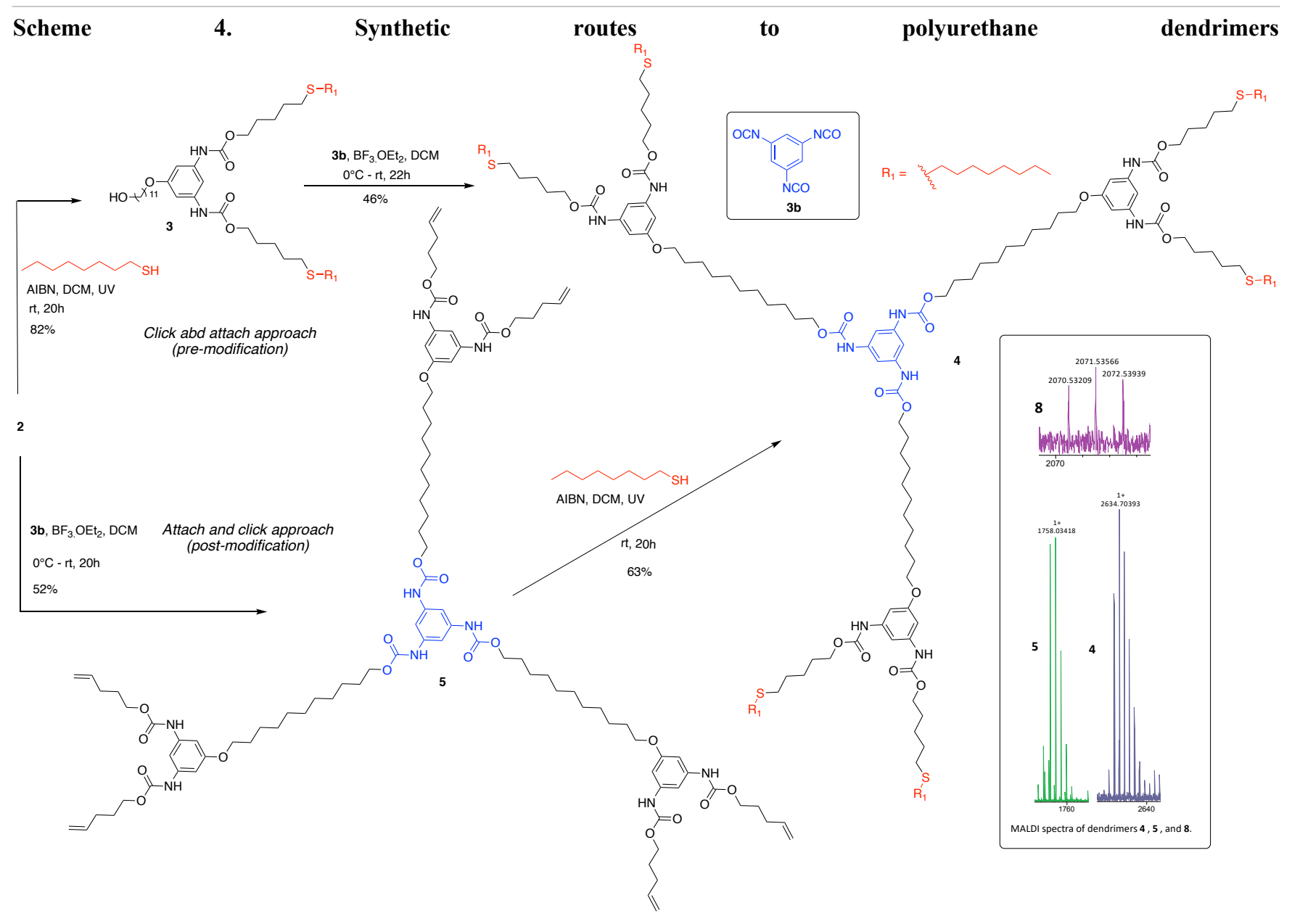

values did not increase the yield of 1 (Table 1; entry 1, 2, and 3) and the base with larger pKa (triethylamine) gave better yields (Table 1; entry 4). This reaction did require a base as depicted by entry 5 in Table 1 , where the yield of $\mathbf{1}$ is negligible in absence of a base. In addition, while good yields were obtained at temperature $85-95^{\circ} \mathrm{C}$, side products were formed in higher amounts at higher temperature. It is noteworthy that one of the side products 1c can be recycled back to branching monomer 1 .

Being a green reaction, thiol-ene click chemistry has been widely used in the efficient growth of dendrimers. ${ }^{23-27} \mathrm{We}$ utilized thiol-ene click here as a tool to ascertain the robustness of monomer 1 towards synthesis of PUDs by functionalizing the dendritic surface via different methods. Accordingly, 4 was synthesized via two different routes as shown in Scheme 4. To accomplish this, 1-octanethiol and 1,3,5triisocyanatobenzene $\mathbf{3 b}$ (preparation in Scheme S3) were selected as thiol-ene click partner and a simple trifunctional core respectively. In its click and attach approach, the wedge 2 was irradiated with 1-octanethiol under UV light in presence of free radical initiator 2,2'- azobis (2-methyl propi- onitrile) (AIBN) to obtain thioether functionalized dendron 3 in high yield (82\%). The convergent synthesis of 4 was accomplished when dendron $\mathbf{3}$ was attached to the core $\mathbf{3 b}$ in presence of Lewis acid $\mathrm{BF}_{3} . \mathrm{OEt}_{2}$. In the attach and click approach, dendron 2 was attached to the core $3 \mathrm{~b}$ under identical conditions to furnish a dendrimer 5 with pentene peripheral groups, which underwent subsequent thiol-ene click with 1-octanethiol under identical reaction conditions to produce dendrimer 4 . Its noticeable that the overall yield of post-modification route is lower because of larger number of reactions required to undergo completion at periphery.

The most powerful feature of convergent synthesis lies on its ability to selectively modify both focal point and chain ends. This strategy allows one to vary the number and type of functional moieties in the resultant dendrimers. In this study, we modified the chain ends without changing its focal point, which in turn resulted in surface modified dendrimer 4. There are two possible approaches for the installation of functionality at the core - introduction of end groups prior to and after the dendritic growth. The structural features of dendron 2 guarantee both pre- and post-modification routes. 


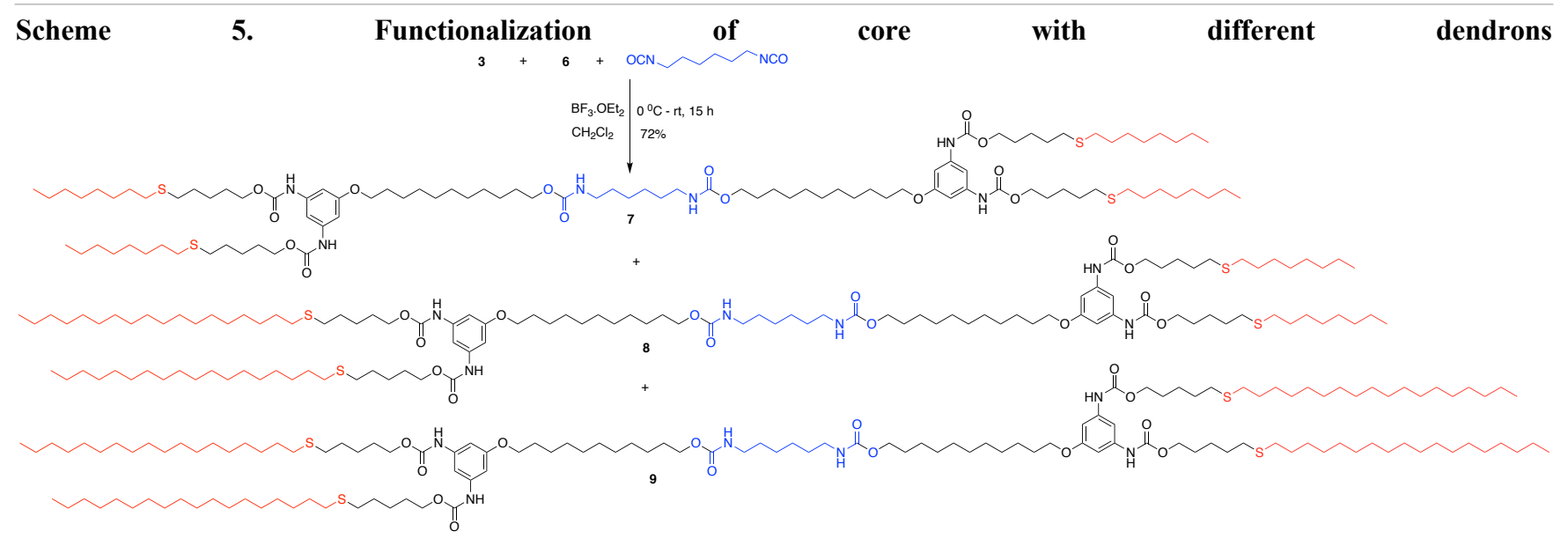

All novel compounds including dendritic wedges and dendrimers were characterized by ${ }^{1} \mathrm{H},{ }^{13} \mathrm{C} \mathrm{NMR}$, and mass spectrometry (HRESI-MS or MALDI-TOF) (details in SI). Figure 1 shows solution state ${ }^{1} \mathrm{H}$ NMR of branching monomers ( 2 and $\mathbf{3}$ ) and dendrimers (4 and 5) in deuterated acetone as a solvent before and after thiol-ene functionalization. Disappearance of peaks with chemical shifts at 5.0 and 5.9 ppm ($\mathrm{CH}=\mathrm{CH}_{2}$ ) (Figure 1a and 1d) and appearance of new peaks at $2.6 \mathrm{ppm}\left(-\mathrm{SCH}_{2^{-}}\right)$and $0.9 \mathrm{ppm}\left(-\mathrm{CH}_{3}\right)$ (Figure 1b and 1c) provided an evidence that thiol-ene click preceded successfully. Moreover, a new peak assigned at $7.5 \mathrm{ppm}$ (aromatic $\mathrm{CH}$ ) (Figure 1c and 1d) furnished further evidence of successful attachment of dendron $\mathbf{2}$ or $\mathbf{3}$ to the trifunctional core.

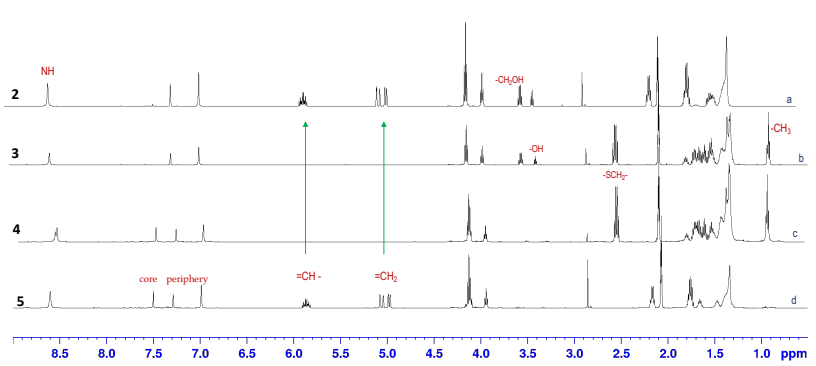

Figure 1. Typical ${ }^{1} \mathrm{H}$ NMR of branching monomers and dendrimers. (a) dendron 2, (b) dendron 3, (c) dendrimer 4, and (d) dendrimer 5. All spectra were taken in $500 \mathrm{MHz}$ spectrometer using $\mathrm{CD}_{3} \mathrm{COCD}_{3}$ as a solvent.

To further exploit the potential of this approach, we embarked on the search for successful functionalization of a different core, hexamethylene diisocyanate (HDI) using two different dendrons (Scheme 5). 1- Octanethiol and 1octadecanethiol clicked dendritic monomers 3 and $\mathbf{6}$ were allowed to attach to the bifunctional core HDI at ambient temperature in presence of $\mathrm{BF}_{3} . \mathrm{OEt}_{2}$ that produced three different dendrimers $(\mathbf{7}, \mathbf{8}$, and 9) (complete structure in SI) as amorphous white solid (72\% yield) including a Janus dendrimer 8. $^{28,29}$ Separation turned out to be simple and convenient with flash chromatography using hexane/ethyl acetate as eluent. At this point, we utilized click and attach approach as overall yield of this approach was higher than at- tach and click approach. It's noteworthy that the proportion of products 7:8:9 is $1: 2: 1$, which in agreement with statistical distribution of their attachment to the core. Thus we anticipate this approach will allow access to hetero or Janus dendrimers. $^{28}$

In summary, we presented thiol-ene click inspired protecting group free approach to the convergent synthesis of polyurethane dendrimers. As a representative of proposed approach, generation one dendrimers were synthesized via click and attach, and attach and click methods under mild conditions. An efficient and robust bifunctional dendron synthesized from a one-pot multicomponent Curtius reaction enabled late stage modification of itself and accompanying dendrimers. Additionally, functionalization of a bifunctional core with two different dendrons furnished a mixture of three dendrimers including a Janus dendrimer. Access to this type of investigation will contribute to concise and versatile synthesis of dendritic macromolecules.

\section{ASSOCIATED CONTENT}

\section{Supporting Information}

The Supporting Information is available free of charge. Experimental procedures, ${ }^{1} \mathrm{H}$ NMR,${ }^{13} \mathrm{C}$ NMR and IR spectra as well as mass spectrometric data of the novel compounds described (PDF)

\section{AUTHOR INFORMATION}

\section{Corresponding Author}

Richard T. Taylor - Department of Chemistry and Biochemistry, Miami University, 501 E High St, Oxford, OH 45056, USA Email: taylorrt@miamioh.edu

\section{Author}

Dhruba P. Poudel - Department of Chemistry and Biochemistry, Miami University, Oxford, OH 45056, USA;

Notes

The authors declare no competing financial interest.

\section{ACKNOWLEDGMENT}

We acknowledge Department of Chemistry and Biochemistry of Miami University for their support. 


\section{REFERENCES}

(1) Tomalia, D. A.; Baker, H.; Hall, M.; Kallos, G.; Martin, S.; Ryder, J.; Smith, P. Dendritic Macromolecules:1 Synthesis of Starburst Dendrimers. Macromolecules 1986, 19 (9), 24662468. https://doi.org/10.1021/ma00163a029.

(2) Sowinska, M.; Urbanczyk-Lipkowska, Z. Advances in the Chemistry of Dendrimers. New J. Chem. 2014, 38 (6), 21682203. https://doi.org/10.1039/c3nj01239e.

(3) Astruc, D.; Chardac, F. Dendritic Catalysts and Dendrimers in Catalysis. Chem. Rev. 2001, 101 (9), 2991-3023. https://doi.org/10.1021/cr010323t.

(4) Majoral, J. P.; Mignani, S. M.; Shi, X.; Rodrigues, J. M.; Muñoz-Fernández, M. Á.; Ceña, V.; Roy, R. Dendrimers towards Translational Nanotherapeutics: Concise Key Step Analysis. Bioconjug. $\quad$ Chem. https://doi.org/10.1021/acs.bioconjchem.0c00395.

(5) Jezek, J.; Reinis M., Sebestik. Biomedical Applications of Peptide-, Glyco-, and Glycopeptide Dendrimers and Analogous Dendrimeric Structures. Springer: Wien, 2012; ISBN: 978-37091-1205-2.

(6) Bruchmann, B. Dendritic Polymers Based on Urethane Chemistry - Syntheses and Applications. Macromol. Mater. Eng. 2007, $292 \quad$ (9), $981-992$. https://doi.org/10.1002/mame.200700119.

(7) Spindler, R.; Fréchet, J. M. J. Two-Step Approach towards the Accelerated Synthesis of Dendritic Macromolecules. J. Chem. Soc. Perkin Trans. 1 1993, No. 8, 913-918. https://doi.org/10.1039/p19930000913.

(8) Reemers, S.; Mourran, A.; Keul, H.; Möller, M. Novel Route to Dendritic Structures and Their Application for Surface Modification. J. Polym. Sci. Part A Polym. Chem. 2006, 44 (4), 1372-1386. https://doi.org/10.1002/pola.21251.

(9) Ali, B. M.; Kumar, K. A.; Nasar, A. S. Fifth-Generation Polyurethane Dendrimers Decorated with Amine, Free Amine, and Blocke Isocyanate End Groups: Synthesis and Electrolytic Performance to Increase the Efficiency of Dye-Sensitized Solar Cell. ChemistrySelect 2014, 5, 12983-12991.

(10) Veerapandian, S.; Nasar, A. S. Amine- and blocked isocyanate-terminated polyurethane dendrimers: integrated synthesis, photophysical proerties, and applications in heat curable system. RSC Adv. 2015, 5, 3800-3806.

(11) Peerlings, H. W. I.; Van Benthem, R. A. T. M.; Meijer, E. W. Fast and Convenient Construction of Carbamate/Urea-Based Dendrimers with a Diisocyanate Building Block. J. Polym. Sci. Part A Polym. Chem. 2001, 39 (18), 3112-3120. https://doi.org/10.1002/pola.1292.

(12) Mohamad Ali, B.; Velavan, B.; Sudhandiran, G.; Sridevi, J.; Sultan Nasar, A. Radical Dendrimers: Synthesis, Anti-Tumor Activity and Enhanced Cytoprotective Performance of TEMPO Free Radical Functionalized Polyurethane Dendrimers. Eur. Polym. J. 2020, 122 (August 2019), 109354. https://doi.org/10.1016/j.eurpolymj.2019.109354.

(13) Hawker, C. J.; Fréchet, J. M. J. Preparation of Polymers with Controlled Molecular Architecture. A New Convergent Approach to Dendritic Macromolecules. J. Am. Chem. Soc. 1990, $112 \quad$ (21), 7638-7647. https://doi.org/10.1021/ja00177a027.

(14) Goodwin, A. P.; Lam, S. S.; Fréchet, J. M. J. Rapid, Efficient Synthesis of Heterofunctional Biodegradable Dendrimers. $J$. Am. Chem. Soc. 2007, 129, 6994-6995.

)

Sikwal, D. R.; Kalhapure, R. S.; Govender, T. An Emerging Class of Amphiphilic Dendrimers for Pharmaceutical and Biomedical Applications: Janus Amphiphilic Dendrimers. Eur. J. Pharm. Sci. 2017, 97, 113-134. https://doi.org/10.1016/j.ejps.2016.11.013. 